Kalpa Publications in Computing
Volume 2, 2017, Pages 62-66
ICRISET2017. International Conference on Re-
search and Innovations in Science, Engineer-
ing \&Technology. Selected Papers in Computing

\title{
An Asymptotic Formula of Modified Family of Positive Linear Operators
}

\author{
Prashantkumar Patel ${ }^{1,2} \&$ Vishnu Narayan Mishra ${ }^{2,3}$ \\ ${ }^{1}$ Department of Mathematics, St. Xavier's College (Autonomous), Ahmedabad-09 (Gujarat), India \\ ${ }^{2}$ Department of Applied Mathematics \& Humanities, Sardar Vallabhbhai National Institute of \\ Technology, Surat-395 007 (Gujarat), India \\ ${ }^{3}$ L. 1627 Awadh Puri Colony Beniganj, Phase -III, Opposite - Industrial Training Institute (I.T.I.), \\ Ayodhya Main Road, Faizabad, 224 001, Uttar Pradesh, India \\ prashant225@gmail.com; vishnu_narayanmishra@yahoo.co.in; vishnunarayanmishra@gmail.com
}

\begin{abstract}
In 2016, Patel and Mishra introduce the operators which is generalization of well-known SzaszMirakyan operators. In this manuscript, we have discussed Voronovskaja asymptotic of Stancu type generalization of the operators defined by Patel and Mishra.

Keywords-Positive linear operators; Asymptotic formula; Sazas-Mirakyan operators
\end{abstract}

\section{Introduction}

Using Lagrange's formula, Patel and Mishra [1] defined the following sequence of positive linear operators, for $f \in C([0, \infty)) ; 0 \leq \mu<1 ; 1<\gamma \leq e$ as

$$
P_{n}^{[\mu, \gamma]}(f ; x)=\sum_{k=0}^{\infty} \omega_{n, \gamma}(k ; n x) f\left(\frac{k}{n}\right)
$$

where

$$
\omega_{n, \gamma}(k, n x)=n x(\log \gamma)^{k}(n x+k \mu)^{k-1} \frac{\gamma^{-(n x+k \mu)}}{(k !)} .
$$

In particular $\gamma=e$, the operators (1) reduce to Jain operators [2]. Also, if $\gamma=e$ and $\mu=0$ then, the operators $P_{n}^{[\mu, \gamma]}$ equal to the classical Szasz-Mirakyan operators [3]. Approximation properties of the Szasz-Mirakyan operators, Jain operators and their generalizations was discussed by many authors. We mention that, approximation properties of the integral generalization of Szasz-Mirakyan operators discussed in $[4,5]$ and integral type generalization of Jain operators discussed in $[6,7,8]$. The generalization of Szasz-Mirakyan operators based on q-integer was established in $[9,10,11]$. This research proved that the Szasz-Mirakyan operators and their generalization have many interesting approximation properties. 
In 1983, the following type generalization of Bernstein polynomial was established by Stancu in [12] and studied the positive linear operators $S_{n}^{\alpha, \beta}: C([0,1]) \rightarrow C([0,1])$ defined for any $f \in C([0,1])$ as follows:

$$
S_{n}^{\alpha, \beta}(f, x)=\sum_{k=0}^{n} p_{(n, k)}(x) f\left(\frac{k+\alpha}{n+\beta}\right), 0 \leq x \leq 1,
$$

where $p_{(n, k)}(x)=\left(\begin{array}{l}n \\ k\end{array}\right) x^{k}(1-x)^{n-k}$ is the Bernstein basis function. After the work of Stancu many researcher work in this direction. The recent work on such type of operators can be found in $[13,14,15$, $16,17,18,19,20,21]$. This motivated us to generalize the operators (1) in the following way, for $f \in$ $C([0, \infty)) ; 0 \leq \mu<1 ; 1<\gamma \leq e, 0 \leq \alpha \leq \beta$ as

$$
P_{n}^{[\mu, \gamma, \alpha, \beta]}(f ; x)=\sum_{k=0}^{\infty} \omega_{n, \gamma}(k ; n x) f\left(\frac{k+\alpha}{n+\beta}\right)
$$

where $\omega_{n, \gamma}(k ; n x)$ as defined in (3). The above generalization known as Stancu type generalization of the operators (1). In particular $\alpha=\beta=0$, the operators (2) reduce to the operators $P_{n}^{[\mu, \gamma]}$.

\section{Some Lemmas}

To discuss moments of the operators (2), we need following lemmas:

Lemma 1([1]). The operators $P_{n}^{[\mu, \gamma]}, n>1$, defined by (1) satisfy the following relations:

1. $P_{n}^{[\mu, \gamma]}(1, x)=1$;

2. $P_{n}^{[\mu, \gamma]}(t, x)=\frac{x \log \gamma}{1-\mu \log \gamma}$;

3. $P_{n}^{[\mu, \gamma]}\left(t^{2}, x\right)=\frac{x^{2}(\log \gamma)^{2}}{(1-\mu \log \gamma)^{2}}+\frac{x \log \gamma}{n(1-\mu \log \gamma)^{3}}$;

4. $P_{n}^{[\mu, \gamma]}\left(t^{3}, x\right)=\frac{x^{3}(\log \gamma)^{3}}{(1-\mu \log \gamma)^{3}}+\frac{3 x^{2}(\log \gamma)^{2}}{n(1-\mu \log \gamma)^{4}}+\frac{x \log \gamma\left(1+2 \mu \log \gamma+{ }^{4}(\log \gamma)^{3}-2 \mu^{4}(\log \gamma)^{4}\right)}{n^{2}(1-\mu \log \gamma)^{5}}$

5. $\quad P_{n}^{[\mu, \gamma]}\left(t^{4}, x\right)=\frac{x^{4}(\log \gamma)^{4}}{(1-\mu \log \gamma)^{4}}+\frac{6 x^{3}(\log \gamma)^{3}}{n(1-\mu \log \gamma)^{5}}+\frac{x^{2}(\log \gamma)^{2}\left(7+8 \mu \log \gamma+{ }^{4}(\log \gamma)^{3}-2 \mu^{4}(\log \gamma)^{4}\right)}{n^{2}(1-\mu \log \gamma)^{6}}$

$$
+\frac{x \log \gamma\left(1+8 \mu \log \gamma+6 \mu^{2}(\log \gamma)^{2}+\left(12 \mu^{4}(\log \gamma)^{3}-16 \mu^{5}(\log \gamma)^{4}+6 \mu^{6}(\log \gamma)^{5}\right)(1-\log \gamma)\right)}{n^{3}(1-\mu \log \gamma)^{7}} .
$$

Lemma 2. The operators $P_{n}^{[\mu, \gamma, \alpha, \beta]}, n>1$, defined by (1) satisfy the following relations:

1. $P_{n}^{[\mu, \gamma, \alpha, \beta]}(1, x)=1$;

2. $P_{n}^{[\mu, \gamma, \alpha, \beta]}(\mathrm{t}, x)=\frac{n x \log \gamma+\alpha(1-\mu \text { lo } \quad)}{(n+\beta)(1-\mu \text { lo })}$;

3. $P_{n}^{[\mu, \gamma, \alpha, \beta]}\left(\mathrm{t}^{2}, x\right)=\frac{n^{2} x^{2}(\log \gamma)^{2}}{(n+\beta)^{2}(1-\mu \log \gamma)^{2}}-\frac{n x \log (1+2 \alpha)}{(n+\beta)^{2}(1-\mu \log \gamma)}+\frac{\alpha^{2}}{(n+\beta)^{2}}$.

Proof. It is clear that $P_{n}^{[\mu, \gamma, \alpha, \beta]}(1, x)=1$. By simple computation, we get 


$$
\begin{gathered}
P_{n}^{[\mu, \gamma, \alpha, \beta]}(\mathrm{t}, x)=\sum_{k=0}^{\infty} \omega_{n, \gamma}(k ; n x)\left(\frac{k+\alpha}{n+\beta}\right)=\frac{n}{n+\beta} P_{n}^{\mu, \gamma}(t, x)+\frac{\alpha}{n+\beta}=\frac{n x \log \gamma+\alpha(1-\mu \log \gamma)}{(n+\beta)(1-\mu \log \gamma)} . \\
\text { Now, } P_{n}^{[\mu, \gamma, \alpha, \beta]}\left(\mathrm{t}^{2}, x\right)=\sum_{k=0}^{\infty} \omega_{n, \gamma}(k ; n x)\left(\frac{k+\alpha}{n+\beta}\right)^{2} \\
=\frac{\mathrm{n}^{2}}{(n+\beta)^{2}} P_{n}^{[\mu, \gamma]}\left(t^{2}, x\right)+\frac{2 \alpha n}{(n+\beta)^{2}} P_{n}^{[\mu, \gamma]}(t, x)+\frac{\alpha^{2}}{(n+\beta)^{2}} \\
=\frac{n^{2} x^{2}(\log \gamma)^{2}}{(n+\beta)^{2}(1-\mu \log \gamma)^{2}}-\frac{n x \log \gamma(1+2 \alpha)}{(n+\beta)^{2}(1-\mu \log \gamma)}+\frac{\alpha^{2}}{(n+\beta)^{2}}
\end{gathered}
$$

we have the desired result.

Remark 1. For all $m \in \mathbb{N}, 0 \leq \alpha \leq \beta$; we have the following recursive relation for the images of the monomials $t^{m}$ under $P_{n}^{[\mu, \gamma, \alpha, \beta]}\left(t^{m}, x\right)$ in terms of $P_{n}^{[\mu, \gamma]}\left(t^{j}, x\right), j=0,1,2, \ldots, m$ as

Remark 2. We have

$$
P_{n}^{[\mu, \gamma, \alpha, \beta]}\left(t^{m}, x\right)=\sum_{j=0}^{m}\left(\begin{array}{c}
m \\
j
\end{array}\right) \frac{n^{j} \alpha^{m-j}}{(n+\beta)^{m}} P_{n}^{[\mu, \gamma]}\left(t^{j}, x\right) .
$$

$$
\begin{gathered}
\Phi_{n}^{[\mu, \gamma, \alpha, \beta]}(x)=P_{n}^{[\mu, \gamma, \alpha, \beta]}(t-x, x)=x\left(\frac{n(\log \gamma-1+\mu \log \gamma)-\beta(1-\mu \log \gamma)}{(n+\beta)(1-\mu \log \gamma)}\right)+\frac{\alpha}{(n+\beta)} \\
\Psi_{n}^{[\mu, \gamma, \alpha, \beta]}(x)=P_{n}^{[\mu, \gamma, \alpha, \beta]}\left((t-x)^{2}, x\right) \\
=x^{2}\left(\frac{(\beta(1-\mu \log \gamma)+n(1-\log \gamma-\mu \log \gamma))^{2}}{(n+\beta)^{2}(1-\mu \log \gamma)^{2}}\right) \\
+x\left(\frac{(n((1+2 \alpha+2 \alpha \mu) \log \gamma-2 \alpha))}{(n+\beta)^{2}(1-\mu \log \gamma)}\right) \\
+x\left(\frac{-2 \alpha \beta(1-\mu \log \gamma)}{(n+\beta)^{2}(1-\mu \log \gamma)}\right)+\frac{\alpha^{2}}{(n+\beta)^{2}} .
\end{gathered}
$$

\section{Voronovskaja Type Theorem}

In this section, we establish the asymptotic formula for the operators $P_{n}^{[\mu, \gamma, \alpha, \beta]}$.

Theorem 1. For $b>0, \mu_{n} \in(0,1)$ such that $n \mu_{n} \rightarrow l \in \mathbb{R}$ and $\gamma_{n} \in(1, e)$ such that $\gamma_{n} \rightarrow e$ (Euler number). Then for every $f \in C([0, b]), f^{\prime}, f^{\prime \prime}$ exists at a fixed point $x \in(0, b)$, we have

$$
\lim _{n \rightarrow \infty} n\left(P_{n}^{\left[\mu_{n}, \gamma_{n}, \alpha, \beta\right]}(f, x)-f(x)\right)=(\alpha+(l-\beta) x) f^{\prime}(x)+\frac{\left(\left(l^{2}+2 \beta\right) x+1\right) x}{2} f^{\prime \prime}(x) .
$$

Proof. Let $x \in(0, b)$ be fixed. From the Taylor's theorem, we may write

$$
f(t)=f(x)+(t-x) f^{\prime}(x)+\frac{1}{2}(t-x)^{2} f^{\prime \prime}(x)+r(t, x)(t-x)^{2},
$$

where $r(t, x)$ is the peano form of the remainder and $\lim _{\mathrm{t} \rightarrow \mathrm{x}} r(t, x)=0$.

Applying $P_{n}^{[\mu, \gamma, \alpha, \beta]}$ on the both side of equation (4), we have 


$$
n\left(P_{n}^{[\mu, \gamma, \alpha, \beta]}(f, x)-f(x)\right)=n f^{\prime}(x) \Phi_{n}^{[\mu, \gamma, \alpha, \beta]}(x)+\frac{1}{2} n f^{\prime \prime}(x) \Psi_{n}^{[\mu, \gamma, \alpha, \beta]}(x) .
$$

In view of Remark 1, we have

$$
\begin{array}{r}
\lim _{n \rightarrow \infty} n \Phi_{n}^{[\mu, \gamma, \alpha, \beta]}(x)=\alpha+(l-\beta) x ; \\
\lim _{n \rightarrow \infty} n \Psi_{n}^{[\mu, \gamma, \alpha, \beta]}(x)=\left(\left(l^{2}+2 \beta\right) x+1\right) x .
\end{array}
$$

Now, we shall show that

$$
\lim _{n \rightarrow \infty} P_{n}^{[\mu, \gamma, \alpha, \beta]}\left(r(t, x)(t-x)^{2}, x\right)=0 .
$$

By using Cauchy-Schwarz inequality, we have

$$
P_{n}^{[\mu, \gamma, \alpha, \beta]}\left(r(t, x)(t-x)^{2}, x\right) \leq\left(P_{n}^{[\mu, \gamma, \alpha, \beta]}\left(r^{2}(t, x), x\right)\right)^{\frac{1}{2}}\left(P_{n}^{[\mu, \gamma, \alpha, \beta]}\left((t-x)^{4}, x\right)\right)^{\frac{1}{2}} .
$$

We observe that $r^{2}(x, x)=0$ and $r^{2}(\cdot, x) \in C([0, b])$. Then, it follows that

$$
\lim _{n \rightarrow \infty} P_{n}^{[\mu, \gamma, \alpha, \beta]}\left(r^{2}(t, x), x\right)=r^{2}(x, x)=0,
$$

in view of the fact that $P_{n}^{[\mu, \gamma, \alpha, \beta]}\left((t-x)^{4}, x\right)=O\left(\frac{1}{n^{2}}\right)$.

Now, from (7) and (8), we obtain

$$
\lim _{n \rightarrow \infty} n P_{n}^{[\mu, \gamma, \alpha, \beta]}\left(r(t, x)(t-x)^{2}, x\right)=0 .
$$

From (5), (6) and (9), we get the required result.

\section{Acknowledgment}

The authors would like to thank the referee for his/her valuable suggestions which improved the paper considerably.

\section{References}

[1] P Patel and V. N. Mishra, "On Generalized Szasz-Mirakyan operators," arXiv preprint arXiv:1508.07896, 2015.

[2] G. C. Jain, "Approximation of functions by a new class of linear operators," J. Aust. Math. Soc., vol. 13, no. 3, pp. 271-276, 1972

[3] O. Szasz, "Generalization of S. Bernsteins polynomials to the infinite interval," J. Res. Natl. Bur. Stand., vol. 45, pp. 239-245, 1950.

[4] O. Duman, M. A. Ozarslan and H. Aktuglu, "Better error Esimation for Szasz-Mirakjan-Beta Operators," Journal of Computational Analysis \& Applications, vol. 10, no. 1, 2008.

[5] H.M. Srivastava and V Gupta , "A certain family of summation-integral type operators," Mathematical and Computer Modelling, vol. 37, no. 12, pp. 1307-1315, 2003.

[6] S. Tarabie, "On Jain-beta linear operators," Appl. Math. Inf. Sci, vol. 6, no. 2, pp. 213-216, 2012. 
[7] P. Patel and V N Mishra, "Jain-Baskakov operators and its different generalization," Acta Mathematica Vietnamica, vol. 4, no. 715-733, p. 40, 2015.

[8] V N Mishra and and P. Patel, "Some approximation properties of modified Jain-Beta operators," Journal of Calculus of Variations, vol. 2013, 2013.

[9] N. I. Mahmudov, "On q-parametric Szasz-Mirakjan operators," Mediterranean journal of mathematics, vol. 7, no. 3, pp. 297-211, 2010.

[10] N. I. Mahmudov, "Approximation properties of complex q-Szasz--Mirakjan operators in compact disks," Computers \& Mathematics with Applications, vol. 60, no. 6, pp. 1784-1791, 2010.

[11] A. Aral, "A generalization of Szasz--Mirakyan operators based on q-integers," Mathematical and Computer Modelling, vol. 47, no. 9, pp. 1052-1062, 2008.

[12] D D Stancu, "Approximation of functions by means of a new generalized," Calcolo, vol. 20, pp. 211-229, 1983.

[13]Büyükyazıc1, İ. and Atakut, Ç, "On Stancu type generalization of q-Baskakov operators," Mathematical and Computer Modelling, vol. 52, no. 5, pp. 752-759, 2010.

[14] Ç Atakut, İ Büyükyazıc1, "Stancu type generalization of the Favard--Szasz operators," Applied Mathematics Letters, vol. 23, no. 12, pp. 1479-1482, 2010.

[15] V. N. Mishra and P. Patel, "Approximation properties of q-Baskakov-Durrmeyer-Stancu operators," Mathematical Sciences, vol. 7, no. 1, pp. 1-12, 2013.

[16] O. Dougru. R M Mohapatra and M Örkcu, "Approximation Properties of Generalized Jain Operators," Filomat, vol. 30, no. 9, pp. 2359-2366, 2016

[17] V. N. Mishra and P. Patel, "The Durrmeyer type modification of the q-Baskakov type operators with two parameter \$lalpha\$ and \$beta\$," Numerical Algorithms, vol. 67, no. 4, pp. 753-769, 2014.

[18] V. N. Mishra and P. Patel, "A short note on approximation properties of Stancu generalization of q-Durrmeyer operators," Fixed point theory and Applications, vol. 2013, no. 1, pp. 1--5, 2013.

[19] V. N.Mishra, K. Khatri and L. N. Mishra, Deepmala, "Inverse result in simultaneous approximation by Baskakov-Durrmeyer-Stancu operators", Journal of Inequalities and Application, vol. 2013, no. 1, pp. 586, 2013.

[20] V. N. Mishra. K. Khatri and L. N. Mishra. On Simultaneous Annroximation for BaskakovDurrmever-Stancu tvpe operators, Journal of Ultra Scientist of Physical Sciences, Vol. 24, No. (3) A, pp. 567-577, 2012.

[21] V. N. Mishra. H. H. Khan. K. Khatri and L. N. Mishra. Hvnergeometric Renresentation for Baskakov-Durrmever-Stancu Tvne Onerators. Bulletin of Mathematical Analysis and Applications, ISSN: 1821-129, Vol. 5, Issue 3, 18-26, 2013. 